\title{
Traumatic injury to pancreatic head with the involvement of common bile duct - a case report
}

\author{
C Lokubalasooriya ${ }^{1}$, WAK Weerawardane ${ }^{2}$, MC Wettasinghe ${ }^{1}$, S Rajapaksha ${ }^{1}$ \\ ${ }^{1}$ Department of Radiology, Teaching Hospital, Anuradhapura \\ ${ }^{2}$ Surgical Unit, Teaching Hospita , Anuradhapura
}

\section{Background \\ Billiary tract injuries are rare following blunt trauma to abdomen but results in serious complications if not identified early and treated promptly.}

We present a case where an adult male was presented with pancreatic head and common bile duct (CBD) injury following blunt trauma to abdomen. While literature states the importance of early surgical interventions, we managed this patient conservatively with use of interventional radiology and patient had an excellent recovery.

\section{Case report}

A twenty five year old male patient presented with one day history of assault to upper abdomen. On presentation he was having severe epigastric pain. Patient was hemodynamically stable. Initial ultrasonography revealed large retroperitoneal hematoma with right renal contusion and some amount of free fluid in the peritoneal cavity.

Patient underwent emergency explorative laparotomy with the suspicion of duodenal injury.

When opened into the peritoneal cavity there was around $400 \mathrm{ml}$ of bile mixed free fluid and a large retroperitoneal hematoma with evidence of right renal contusion. Further exploration revealed injury to the head of the. There was no evidence of duodenal injury. Abdomen was closed after insertion of two drains to the peritoneal cavity with one in the duodenal area and the other in the pelvis. There was continuous bile stained fluid draining from the drain in the duodenal area. Subcutaneous octreotide was given for 15 days. During this period very high amylase levels were detected in drained fluid for three weeks and gradually it got lesser. Comparatively the serum amylase levels were elevated for a lesser degree. Patient was on total parenteral nutrition for 12 days.

Patient developed jaundice by $5^{\text {th }}$ day. Ultrasound scan abdomen revealed normal sized CBD. Opacified billiary system was seen when non ionic contrast was injected through the pelvic drain indicating free communication of the CBD with the peritoneal cavity (Figure 1 and 2).

There was continuous bile stained fluid draining from the drain in the duodenal area. He underwent ultrasound guided CBD stenting (Figure 3). Bile stained drainage gradually got lesser and after 17 days following the trauma it was stopped.

He developed dyspnoea while in the ward on post op day 25 and was observed in the ICU for 7 days. 


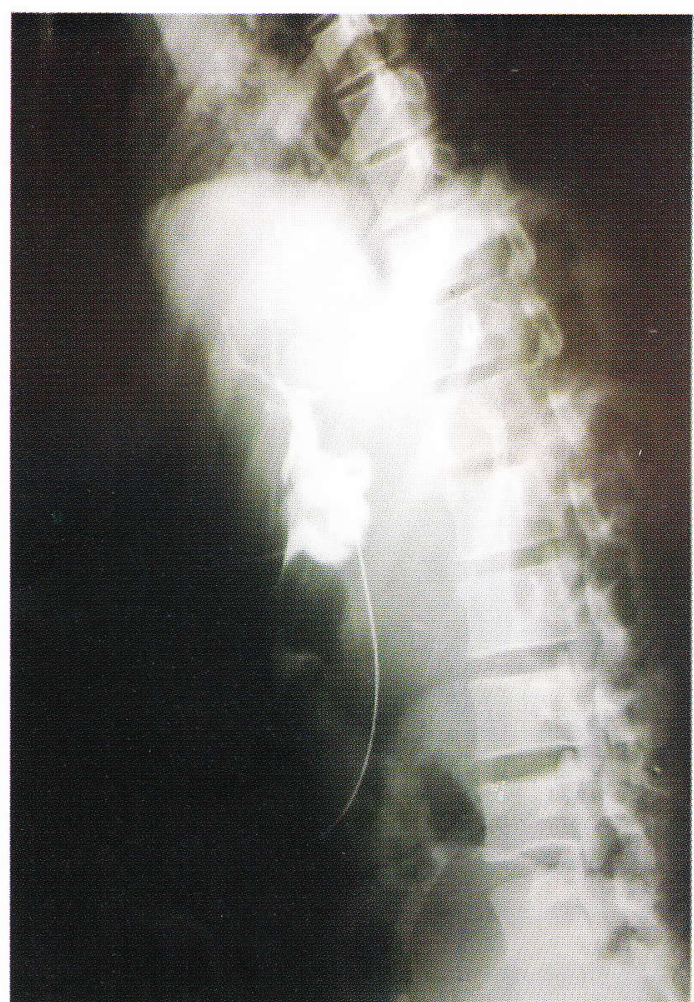

Figure 1

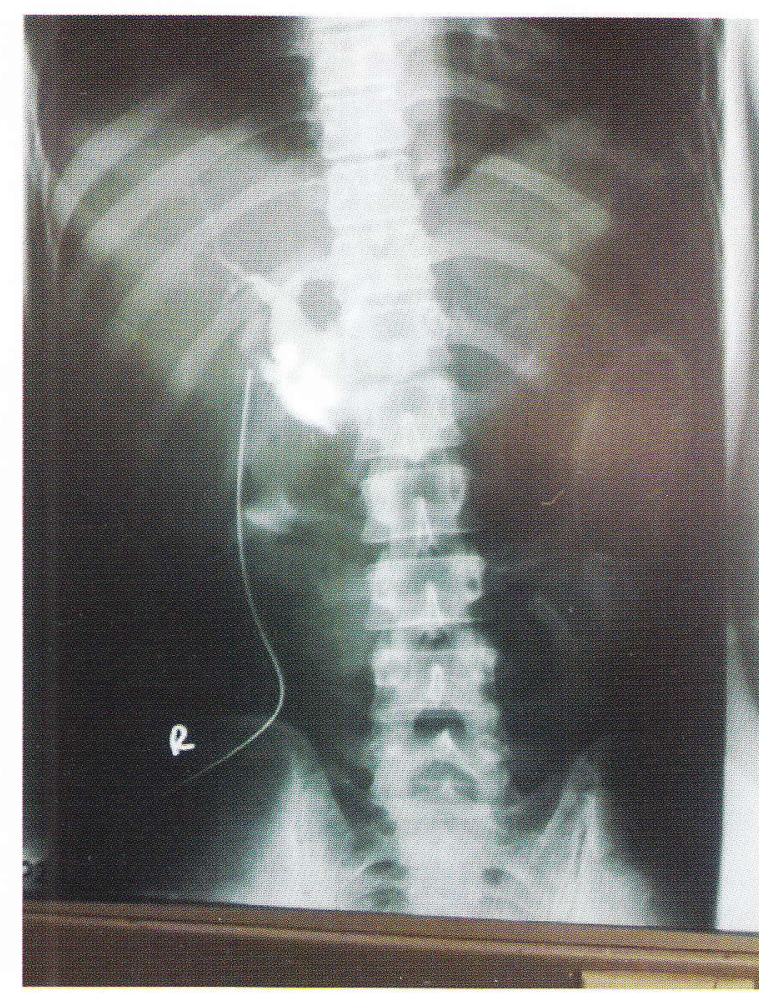

Figure 2

(21.04.2011)

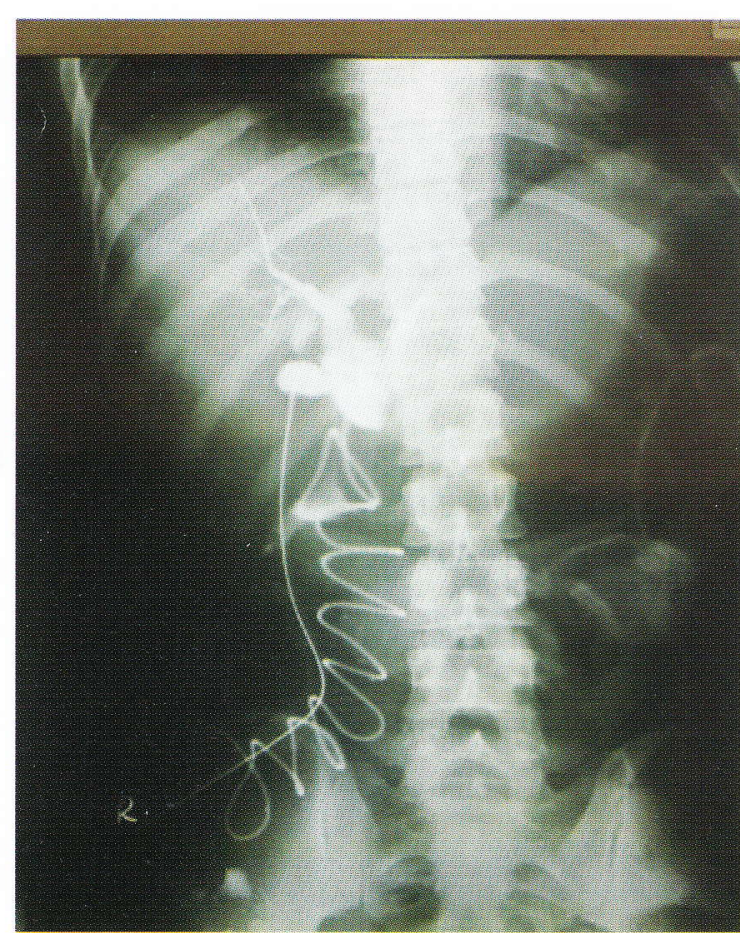

Figure 3

Ultrasound scan abdomen done at this stage revealed two large subdiaphragmatic collections. Ultrasound guided drainage of these collections were done on several occasions. Tubogram was performed when the drainage fluid became free of bile by injecting non ionic contrast media through CBD stent and contrast entered duodenum with no spillage (Figure 4). During the hospital stay he developed lower respiratory tract infection where the sputum culture became positive for pseudomonas. He developed peritonitis and pseudomonas were isolated from the drain fluid.

Afterwards patient had an uneventful recovery.

CBD stenting -07.05.2011 


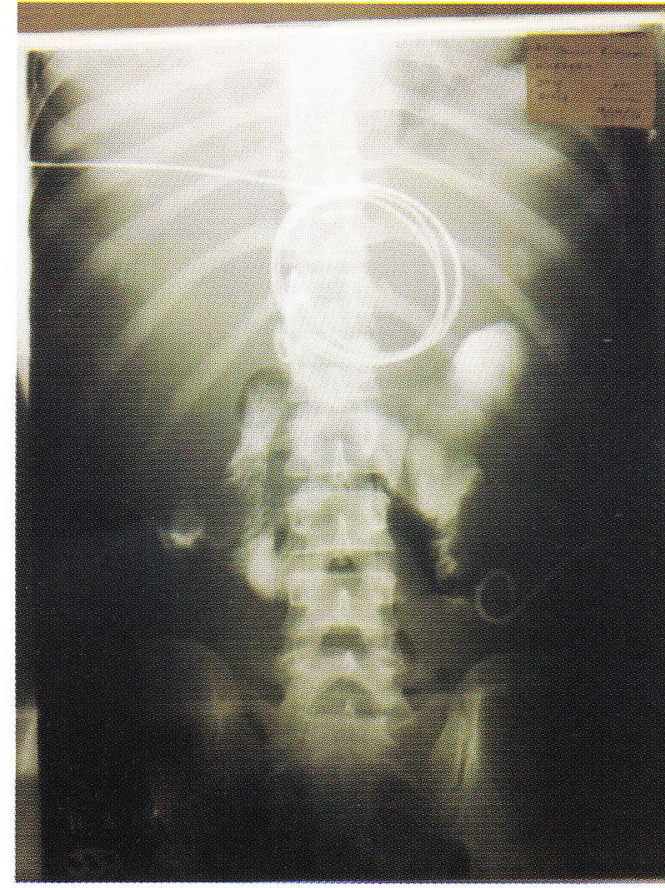

Figure 4

Tubogrham- bile is draining to duodenum $18 / 05 / 2011$

\section{Discussion}

We present this case history to highlight the importance of timely radiological interventions to prevent the hazardous effects of extrahepatic bile duct injuries, without surgical correction of the damaged CBDExtrahepatic billiary tract injuries are rare after the blunt trauma of the abdomen. The gallbladder is the most commonly injured part of the extrahepatic biliary tract, followed by common bile duct, confluence of hepatic ducts and then right or left hepatic duct ${ }^{1}$.

Early diagnosis of biliary tract injuries always required a high index of clinical suspicion guided by timely utilization of diagnosis modalities including peritoneal tap, ultrasonography,

CT scan, HIDA scan and ERCP. ${ }^{1}$

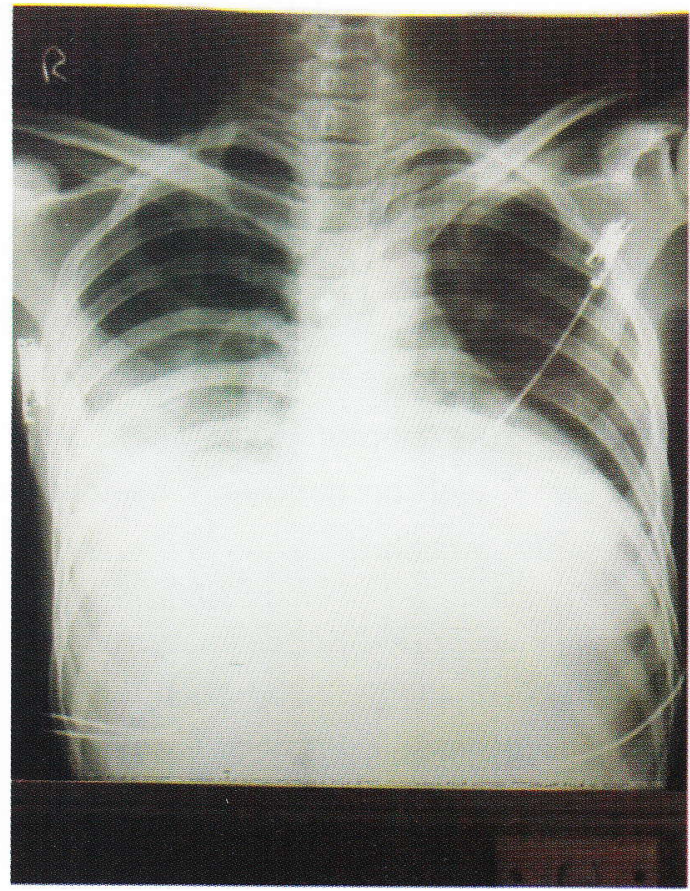

Chest Xray.

Patient transferred to ICU (12/05/2011)

dyspnoeic ( RR -40) PR-132 SPo2-80\% high fever spike

Ultrasound can show the presence of free abdominal fluid and, as such, provide collateral evidence in cases of suspected pancreatic injury. The assessment of the pancreas per se is difficult because of its retroperitoneal position and the presence of overlying transverse colonic gas?

Initial peritoneal fluid amylase was high in this patient but gradually it got lesser.

Serum amylase was initially considered a reliable indicator of pancreatic trauma. Further studies demonstrated that elevation of amylase in neither serum nor peritoneal lavage fluid was sensitive or specific in the diagnosis of pancreatic injury ${ }^{2}$

Subcutaneous octreotide was give to reduce complications following pancreatic injuries. 
Literature has shown tha6t the use of octreotide minimized the complications following pancreatic injuries

Pancreatic complications occurred frequently (21\%) following pancreatic trauma and resulted in significant morbidity. In this nonrandomized series of patients with equivalent ATI, ISS, and pancreatic grade injuries, the prophylactic use of octreotide was associated with no pancreatic complications and no negative sequelae ${ }^{3}$

A minimally invasive, multidisciplinary approach to traumatic bile leaks, as an alternative to open surgery, is practical and safe. It requires flexibility, particularly if the diagnosis has been delayed, and may still involve a prolonged hospital stay ${ }^{4}$

\section{References}

1. Mohammed S. Mallick, Abdulrahman A. AlBassam, Ahmed A. Boukai. Non operative management of blunt bile duct injuries in children.Saudi Med J 2002; Vol. 23 (3): 341-344.

2. Degiannis. E, Glapa M, Loukogeorgakis S.P, Smith M.D. Management of pancreatic trauma. Injury, Int. J. Care Injured (2008) 39, 21-29.

3. Amirata E, Livingston DH, Elcavage J. Octreotide acetate decreases pancreatic complications after pancreatic trauma. Am J Surg. 1994 Oct;168(4):345-7.
Percutaneous transhepatic cholangiography is a safe and accurate procedure that is an important step in the workup of jaundice following blunt trauma.

Progressive interventional radiology and endoscopy now permits non-operative anagement

by establishing efficient percutaneous drainage in leak vicinity and accurate visualizing of the site of biliary injury and placing endoprosthesis to reduce the physiological intrabiliary pressure and to bridge the biliary defect. ${ }^{1}$

Thus, with the rational use of limited resources available, prompt timely radiological intervention lead to the uneventful recovery of the patient.

4. Castagnetti M, Houben C, Patel S, Devlin J, Harrison P, Karani J, Heaton N, Davenport M.

Minimally invasive management of bile leaks after blunt liver trauma in children.Journal of Pediatric Surgery Volume 41, Issue 9, September 2006, Pages 1539-1544

5. Burt TB, Nelson JA. Extrahepatic biliary duct trauma- A spectrum of injuries. West J Med, Apr 1981 134:283-289.

\section{Authors}

- C Lokubalasooriya

Consultant Radiologist

Department of Radiology, Teaching Hospital, Anuradhapura

\section{- WAK Weerawardane}

Consultant Surgeon

Surgical Unit, Teaching Hospita , Anuradhapura

- MC Wettasinghe* S Rajapaksha*

\author{
* Senior House Officers \\ Department of Radiology, Teaching Hospital, Anuradhapura
}

